\title{
\begin{tabular}{l|l} 
Mitraries & DSpace@MIT
\end{tabular}
}

\author{
MIT Open Access Articles
}

Direct Measurement of the Total Decay Width of the Top Quark

The MIT Faculty has made this article openly available. Please share how this access benefits you. Your story matters.

Citation: Aaltonen, T., S. Amerio, D. Amidei, A. Anastassov, A. Annovi, J. Antos, G. Apollinari, et al. "Direct Measurement of the Total Decay Width of the Top Quark." Physical Review Letters 111, no. 20 (November 2013). (C) 2013 American Physical Society

As Published: http://dx.doi.org/10.1103/PhysRevLett.111.202001

Publisher: American Physical Society

Persistent URL: http://hdl.handle.net/1721.1/84931

Version: Final published version: final published article, as it appeared in a journal, conference proceedings, or other formally published context

Terms of Use: Article is made available in accordance with the publisher's policy and may be subject to US copyright law. Please refer to the publisher's site for terms of use. 


\section{Direct Measurement of the Total Decay Width of the Top Quark}

T. Aaltonen, ${ }^{22}$ S. Amerio, ${ }^{41,40}$ D. Amidei,${ }^{32}$ A. Anastassov, ${ }^{16, w}$ A. Annovi, ${ }^{18}$ J. Antos, ${ }^{13}$ G. Apollinari, ${ }^{16}$ J. A. Appel, ${ }^{16}$ T. Arisawa ${ }^{63}$ A. Artikov, ${ }^{14}$ J. Asaadi, ${ }^{55}$ W. Ashmanskas, ${ }^{16}$ B. Auerbach, ${ }^{2}$ A. Aurisano, ${ }^{55}$ F. Azfar, ${ }^{39}$ W. Badgett, ${ }^{16}$ T. Bae, ${ }^{26}$ A. Barbaro-Galtieri, ${ }^{27}$ V.E. Barnes,${ }^{50}$ B. A. Barnett, ${ }^{24}$ P. Barria,${ }^{45,43}$ P. Bartos,${ }^{13}$ M. Bauce,${ }^{41,40}$ F. Bedeschi, ${ }^{43}$ S. Behari, ${ }^{16}$ G. Bellettini,${ }^{44,43}$ J. Bellinger, ${ }^{65}$ D. Benjamin, ${ }^{15}$ A. Beretvas, ${ }^{16}$ A. Bhatti, ${ }^{52}$ K. R. Bland, ${ }^{5}$ B. Blumenfeld, ${ }^{24}$ A. Bocci, ${ }^{15}$ A. Bodek,${ }^{51}$ D. Bortoletto,${ }^{50}$ J. Boudreau, ${ }^{49}$ A. Boveia, ${ }^{12}$ L. Brigliadori, ${ }^{7,6}$ C. Bromberg, ${ }^{33}$ E. Brucken, ${ }^{22}$ J. Budagov, ${ }^{14}$ H. S. Budd,${ }^{51}$ K. Burkett, ${ }^{16}$ G. Busetto, ${ }^{41,40}$ P. Bussey, ${ }^{20}$ P. Butti,${ }^{44,43}$ A. Buzatu, ${ }^{20}$ A. Calamba, ${ }^{11}$ S. Camarda, ${ }^{4}$ M. Campanelli, ${ }^{29}$ F. Canelli, ${ }^{12, \mathrm{dd}}$ B. Carls ${ }^{23}$ D. Carlsmith, ${ }^{65}$ R. Carosi, ${ }^{43}$ S. Carrillo, ${ }^{17, m}$ B. Casal, ${ }^{10, k}$ M. Casarsa ${ }^{56}$ A. Castro, ${ }^{7,6}$ P. Catastini,${ }^{21}$ D. Cauz,${ }^{57,58,56}$ V. Cavaliere, ${ }^{23}$ M. Cavalli-Sforza, ${ }^{4}$ A. Cerri, ${ }^{27, f}$ L. Cerrito, ${ }^{29, r}$ Y.C. Chen, ${ }^{1}$ M. Chertok, ${ }^{8}$ G. Chiarelli, ${ }^{43}$ G. Chlachidze, ${ }^{16}$ K. Cho, ${ }^{26}$ D. Chokheli, ${ }^{14}$ A. Clark, ${ }^{19}$ C. Clarke, ${ }^{64}$ M.E. Convery, ${ }^{16}$ J. Conway, ${ }^{8}$ M. Corbo, ${ }^{16, z}$ M. Cordelli, ${ }^{18}$ C. A. Cox,${ }^{8}$ D. J. Cox,${ }^{8}$ M. Cremonesi, ${ }^{43}$ D. Cruz,${ }^{55}$ J. Cuevas, ${ }^{10, y}$ R. Culbertson, ${ }^{16}$ N. d'Ascenzo, ${ }^{16, v}$ M. Datta, ${ }^{16, g g}$ P. de Barbaro, ${ }^{51}$ L. Demortier,${ }^{52}$ M. Deninno, ${ }^{6}$ M. D’Errico, ${ }^{41,40}$ F. Devoto, ${ }^{22}$ A. Di Canto,${ }^{44,43}$ B. Di Ruzza, ${ }^{16, q}$ J. R. Dittmann, ${ }^{5}$ S. Donati, ${ }^{44,43}$ M. D'Onofrio, ${ }^{28}$ M. Dorigo, ${ }^{59,56}$ A. Driutti, ${ }^{57,58,56}$ K. Ebina, ${ }^{63}$ R. Edgar, ${ }^{32}$ A. Elagin, ${ }^{55}$ R. Erbacher, ${ }^{8}$ S. Errede, ${ }^{23}$ B. Esham, ${ }^{23}$ S. Farrington, ${ }^{39}$ J. P. Fernández Ramos,${ }^{30}$ R. Field, ${ }^{17}$ G. Flanagan, ${ }^{16, t}$ R. Forrest, ${ }^{8}$ M. Franklin, ${ }^{21}$ J. C. Freeman, ${ }^{16}$ H. Frisch, ${ }^{12}$ Y. Funakoshi, ${ }^{63}$ C. Galloni,${ }^{44,43}$ A. F. Garfinkel,${ }^{50}$ P. Garosi, ${ }^{45,43}$ H. Gerberich,${ }^{23}$ E. Gerchtein, ${ }^{16}$ S. Giagu, ${ }^{53}$

V. Giakoumopoulou, ${ }^{3}$ K. Gibson, ${ }^{49}$ C. M. Ginsburg, ${ }^{16}$ N. Giokaris, ${ }^{3}$ P. Giromini, ${ }^{18}$ G. Giurgiu,${ }^{24}$ V. Glagolev, ${ }^{14}$ D. Glenzinski, ${ }^{16}$ M. Gold ${ }^{35}$ D. Goldin, ${ }^{55}$ A. Golossanov, ${ }^{16}$ G. Gomez, ${ }^{10}$ G. Gomez-Ceballos, ${ }^{31}$ M. Goncharov, ${ }^{31}$ O. González López, ${ }^{30}$ I. Gorelov, ${ }^{35}$ A. T. Goshaw, ${ }^{15}$ K. Goulianos, ${ }^{52}$ E. Gramellini, ${ }^{6}$ S. Grinstein, ${ }^{4}$ C. Grosso-Pilcher, ${ }^{12}$ R. C. Group ${ }^{62,16}$ J. Guimaraes da Costa, ${ }^{21}$ S. R. Hahn, ${ }^{16}$ J. Y. Han, ${ }^{51}$ F. Happacher, ${ }^{18}$ K. Hara ${ }^{60}$ M. Hare, ${ }^{61}$ R. F. Harr, ${ }^{64}$

T. Harrington-Taber, ${ }^{16, n}$ K. Hatakeyama, ${ }^{5}$ C. Hays, ${ }^{39}$ J. Heinrich, ${ }^{42}$ M. Herndon, ${ }^{65}$ A. Hocker, ${ }^{16}$ Z. Hong, ${ }^{55}$ W. Hopkins, ${ }^{16,9}$ S. Hou, ${ }^{1}$ R. E. Hughes,${ }^{36}$ U. Husemann, ${ }^{66}$ M. Hussein, ${ }^{33, b b}$ J. Huston, ${ }^{33}$ G. Introzzi, ${ }^{47,48,43}$ M. Iori, ${ }^{54,53}$ A. Ivanov, ${ }^{8, p}$ E. James, ${ }^{16}$ D. Jang, ${ }^{11}$ B. Jayatilaka, ${ }^{16}$ E. J. Jeon, ${ }^{26}$ S. Jindariani, ${ }^{16}$ M. Jones, ${ }^{50}$ K. K. Joo, ${ }^{26}$ S. Y. Jun, ${ }^{11}$ T. R. Junk, ${ }^{16}$ M. Kambeitz, ${ }^{25}$ T. Kamon, ${ }^{26,55}$ P.E. Karchin, ${ }^{64}$ A. Kasmi, ${ }^{5}$ Y. Kato, ${ }^{38, o}$ W. Ketchum, ${ }^{12, h h}$ J. Keung, ${ }^{42}$

B. Kilminster, ${ }^{16, d d}$ D. H. Kim, ${ }^{26}$ H. S. Kim, ${ }^{26}$ J.E. Kim, ${ }^{26}$ M. J. Kim, ${ }^{18}$ S. H. Kim, ${ }^{60}$ S. B. Kim, ${ }^{26}$ Y. J. Kim, ${ }^{26}$ Y. K. Kim, ${ }^{12}$ N. Kimura, ${ }^{63}$ M. Kirby, ${ }^{16}$ K. Knoepfel,${ }^{16}$ K. Kondo, ${ }^{63, a}$ D. J. Kong, ${ }^{26}$ J. Konigsberg, ${ }^{17}$ A. V. Kotwal, ${ }^{15}$ M. Kreps, ${ }^{25}$ J. Kroll, ${ }^{42}$ M. Kruse, ${ }^{15}$ T. Kuhr, ${ }^{25}$ M. Kurata, ${ }^{60}$ A. T. Laasanen, ${ }^{50}$ S. Lammel, ${ }^{16}$ M. Lancaster, ${ }^{29}$

K. Lannon, ${ }^{36, x}$ G. Latino, ${ }^{45,43}$ H. S. Lee, ${ }^{26}$ J. S. Lee, ${ }^{26}$ S. Leo, ${ }^{43}$ S. Leone, ${ }^{43}$ J. D. Lewis, ${ }^{16}$ A. Limosani, ${ }^{15, s}$ E. Lipeles, ${ }^{42}$ A. Lister, ${ }^{19, b}$ H. Liu, ${ }^{62}$ Q. Liu, ${ }^{50}$ T. Liu, ${ }^{16}$ S. Lockwitz, ${ }^{66}$ A. Loginov, ${ }^{66}$ D. Lucchesi, ${ }^{41,40}$ A. Lucà, ${ }^{18}$ J. Lueck, ${ }^{25}$ P. Lujan, ${ }^{27}$ P. Lukens, ${ }^{16}$ G. Lungu, ${ }^{52}$ J. Lys, ${ }^{27}$ R. Lysak, ${ }^{13, e}$ R. Madrak,${ }^{16}$ P. Maestro, ${ }^{45,43}$ S. Malik, ${ }^{52}$ G. Manca, ${ }^{28, c}$ A. Manousakis-Katsikakis, ${ }^{3}$ L. Marchese, ${ }^{6, i i}$ F. Margaroli, ${ }^{53}$ P. Marino,${ }^{46,43}$ M. Martínez, ${ }^{4}$ K. Matera, ${ }^{23}$ M. E. Mattson, ${ }^{64}$ A. Mazzacane, ${ }^{16}$ P. Mazzanti, ${ }^{6}$ R. McNulty ${ }^{28, j}$ A. Mehta, ${ }^{28}$ P. Mehtala ${ }^{22}$ C. Mesropian, ${ }^{52}$ T. Miao, ${ }^{16}$ D. Mietlicki, ${ }^{32}$ A. Mitra, ${ }^{1}$ H. Miyake, ${ }^{60}$ S. Moed, ${ }^{16}$ N. Moggi, ${ }^{6}$ C. S. Moon, ${ }^{16, z}$ R. Moore, ${ }^{16, e e, f f}$ M. J. Morello, ${ }^{46,43}$ A. Mukherjee, ${ }^{16}$ Th. Muller, ${ }^{25}$ P. Murat, ${ }^{16}$ M. Mussini, ${ }^{7,6}$ J. Nachtman, ${ }^{16, n}$ Y. Nagai ${ }^{60}$ J. Naganoma, ${ }^{63}$ I. Nakano, ${ }^{37}$ A. Napier, ${ }^{61}$ J. Nett,${ }^{55}$ C. Neu, ${ }^{62}$ T. Nigmanov, ${ }^{49}$ L. Nodulman, ${ }^{2}$ S. Y. Noh, ${ }^{26}$ O. Norniella, ${ }^{23}$ L. Oakes,${ }^{39}$ S. H. Oh, ${ }^{15}$ Y. D. Oh,${ }^{26}$ I. Oksuzian, ${ }^{62}$ T. Okusawa ${ }^{38}$ R. Orava,${ }^{22}$ L. Ortolan, ${ }^{4}$ C. Pagliarone, ${ }^{56}$ E. Palencia, ${ }^{10, f}$ P. Palni, ${ }^{35}$ V. Papadimitriou, ${ }^{16}$ W. Parker, ${ }^{65}$ G. Pauletta, ${ }^{57,58,56}$ M. Paulini, ${ }^{11}$ C. Paus,${ }^{31}$ T. J. Phillips, ${ }^{15}$ G. Piacentino, ${ }^{43}$ E. Pianori, ${ }^{42}$ J. Pilot, ${ }^{8}$ K. Pitts,${ }^{23}$ C. Plager, ${ }^{9}$ L. Pondrom, ${ }^{65}$ S. Poprocki, ${ }^{16, \mathrm{~g}}$ K. Potamianos, ${ }^{27}$ A. Pranko, ${ }^{27}$ F. Prokoshin,,${ }^{14, \text { aa }}$ F. Ptohos, ${ }^{18, \mathrm{~h}}$ G. Punzi, ${ }^{44,43}$ N. Ranjan, ${ }^{50}$ I. Redondo Fernández, ${ }^{30}$ P. Renton, ${ }^{39}$ M. Rescigno, ${ }^{53}$ F. Rimondi, ${ }^{6, a}$ L. Ristori, ${ }^{43,16}$ A. Robson, ${ }^{20}$ T. Rodriguez, ${ }^{42}$ S. Rolli, ${ }^{61, i}$ M. Ronzani, ${ }^{44,43}$ R. Roser,${ }^{16}$ J. L. Rosner, ${ }^{12}$ F. Ruffini, ${ }^{45,43}$ A. Ruiz, ${ }^{10}$ J. Russ, ${ }^{11}$ V. Rusu, ${ }^{16}$ W. K. Sakumoto, ${ }^{51}$ Y. Sakurai, ${ }^{63}$ L. Santi, ${ }^{57,58,56}$ K. Sato, ${ }^{60}$ V. Saveliev, ${ }^{16, v}$ A. Savoy-Navarro, ${ }^{16, z}$ P. Schlabach ${ }^{16}$ E. E. Schmidt, ${ }^{16}$ T. Schwarz, ${ }^{32}$ L. Scodellaro, ${ }^{10}$ F. Scuri, ${ }^{43}$ S. Seidel,,${ }^{35}$ Y. Seiya, ${ }^{38}$ A. Semenov, ${ }^{14}$ F. Sforza, ${ }^{44,43}$ S. Z. Shalhout, ${ }^{8}$ T. Shears ${ }^{28}$ P. F. Shepard, ${ }^{49}$ M. Shimojima, ${ }^{60, u}$ M. Shochet, ${ }^{12}$ I. Shreyber-Tecker, ${ }^{34}$ A. Simonenko, ${ }^{14}$ K. Sliwa, ${ }^{61}$ J. R. Smith,${ }^{8}$ F. D. Snider,${ }^{16}$ H. Song, ${ }^{49}$ V. Sorin, ${ }^{4}$ R. St. Denis, ${ }^{20}$ M. Stancari, ${ }^{16}$ D. Stentz, ${ }^{16, w}$ J. Strologas, ${ }^{35}$ Y. Sudo, ${ }^{60}$ A. Sukhanov, ${ }^{16}$ I. Suslov, ${ }^{14}$ K. Takemasa,${ }^{60}$ Y. Takeuchi, ${ }^{60}$ J. Tang, ${ }^{12}$ M. Tecchio, ${ }^{32}$ P. K. Teng, ${ }^{1}$ J. Thom, ${ }^{16, g}$ E. Thomson, ${ }^{42}$ V. Thukral,${ }^{55}$ D. Toback,${ }^{55}$ S. Tokar, ${ }^{13}$ K. Tollefson, ${ }^{33}$ T. Tomura,${ }^{60}$ D. Tonelli,,${ }^{16, f}$ S. Torre, ${ }^{18}$ D. Torretta, ${ }^{16}$ P. Totaro, ${ }^{40} \mathrm{M}$. Trovato, ${ }^{46,43} \mathrm{~F}$. Ukegawa, ${ }^{60} \mathrm{~S}$. Uozumi, ${ }^{26}$ F. Vázquez, ${ }^{17, \mathrm{~m}} \mathrm{G}$. Velev, ${ }^{16} \mathrm{C}$. Vellidis, ${ }^{16}$ C. Vernieri, ${ }^{46,43}$ M. Vidal, ${ }^{50}$ R. Vilar, ${ }^{10}$ J. Vizán,,${ }^{10, c c}$ M. Vogel,${ }^{35}$ G. Volpi,${ }^{18}$ P. Wagner, ${ }^{42}$ R. Wallny, ${ }^{16, k}$ S. M. Wang, ${ }^{1}$ D. Waters ${ }^{29}$ W. C. Wester III, ${ }^{16}$ D. Whiteson, ${ }^{42, d}$ A. B. Wicklund, ${ }^{2}$ S. Wilbur, ${ }^{8}$ H. H. Williams,${ }^{42}$ J. S. Wilson, ${ }^{32}$ 
P. Wilson, ${ }^{16}$ B. L. Winer, ${ }^{36}$ P. Wittich, ${ }^{16, g}$ S. Wolbers, ${ }^{16} \mathrm{H}$. Wolfe, ${ }^{36} \mathrm{~T}$. Wright,${ }^{32} \mathrm{X}$. Wu, ${ }^{19} \mathrm{Z} . \mathrm{Wu},{ }^{5} \mathrm{~K}$. Yamamoto, ${ }^{38}$ D. Yamato, ${ }^{38}$ T. Yang, ${ }^{16}$ U. K. Yang, ${ }^{26}$ Y. C. Yang, ${ }^{26}$ W.-M. Yao, ${ }^{27}$ G.P. Yeh, ${ }^{16}$ K. Yi, ${ }^{16, n}$ J. Yoh, ${ }^{16}$ K. Yorita, ${ }^{63}$ T. Yoshida, ${ }^{38,1}$ G. B. Yu, ${ }^{15}$ I. Yu, ${ }^{26}$ A. M. Zanetti, ${ }^{56}$ Y. Zeng, ${ }^{15}$ C. Zhou, ${ }^{15}$ and S. Zucchelli ${ }^{7,6}$

(CDF Collaboration)

\author{
${ }^{1}$ Institute of Physics, Academia Sinica, Taipei, Taiwan 11529, Republic of China \\ ${ }^{2}$ Argonne National Laboratory, Argonne, Illinois 60439, USA \\ ${ }^{3}$ University of Athens, 15771 Athens, Greece \\ ${ }^{4}$ Institut de Fisica d'Altes Energies, ICREA, Universitat Autonoma de Barcelona, E-08193, Bellaterra (Barcelona), Spain \\ ${ }^{5}$ Baylor University, Waco, Texas 76798, USA \\ ${ }^{6}$ Istituto Nazionale di Fisica Nucleare Bologna, I-40127 Bologna, Italy \\ ${ }^{7}$ University of Bologna, I-40127 Bologna, Italy \\ ${ }^{8}$ University of California, Davis, Davis, California 95616, USA \\ ${ }^{9}$ University of California, Los Angeles, Los Angeles, California 90024, USA \\ ${ }^{10}$ Instituto de Fisica de Cantabria, CSIC-University of Cantabria, 39005 Santander, Spain \\ ${ }^{11}$ Carnegie Mellon University, Pittsburgh, Pennsylvania 15213, USA \\ ${ }^{12}$ Enrico Fermi Institute, University of Chicago, Chicago, Illinois 60637, USA \\ ${ }^{13}$ Comenius University, 84248 Bratislava, Slovakia; Institute of Experimental Physics, 04001 Kosice, Slovakia \\ ${ }^{14}$ Joint Institute for Nuclear Research, RU-141980 Dubna, Russia \\ ${ }^{15}$ Duke University, Durham, North Carolina 27708, USA \\ ${ }^{16}$ Fermi National Accelerator Laboratory, Batavia, Illinois 60510, USA \\ ${ }^{17}$ University of Florida, Gainesville, Florida 32611, USA \\ ${ }^{18}$ Laboratori Nazionali di Frascati, Istituto Nazionale di Fisica Nucleare, I-00044 Frascati, Italy \\ ${ }^{19}$ University of Geneva, CH-1211 Geneva 4, Switzerland \\ ${ }^{20}$ Glasgow University, Glasgow G12 8QQ, United Kingdom \\ ${ }^{21}$ Harvard University, Cambridge, Massachusetts 02138, USA \\ ${ }^{22}$ Division of High Energy Physics, Department of Physics, University of Helsinki, FIN-00014 Helsinki, Finland; \\ Helsinki Institute of Physics, FIN-00014, Helsinki, Finland \\ ${ }^{23}$ University of Illinois, Urbana, Illinois 61801, USA \\ ${ }^{24}$ The Johns Hopkins University, Baltimore, Maryland 21218, USA \\ ${ }^{25}$ Institut für Experimentelle Kernphysik, Karlsruhe Institute of Technology, D-76131 Karlsruhe, Germany \\ ${ }^{26}$ Center for High Energy Physics: Kyungpook National University, Daegu 702-701, Korea; \\ Seoul National University, Seoul 151-742, Korea; \\ Sungkyunkwan University, Suwon 440-746, Korea; \\ Korea Institute of Science and Technology Information, Daejeon 305-806, Korea; \\ Chonnam National University, Gwangju 500-757, Korea; \\ Chonbuk National University, Jeonju 561-756, Korea; \\ Ewha Womans University, Seoul, 120-750, Korea \\ ${ }^{27}$ Ernest Orlando Lawrence Berkeley National Laboratory, Berkeley, California 94720, USA \\ ${ }^{28}$ University of Liverpool, Liverpool L69 7ZE, United Kingdom \\ ${ }^{29}$ University College London, London WC1E 6BT, United Kingdom \\ ${ }^{30}$ Centro de Investigaciones Energeticas Medioambientales y Tecnologicas, E-28040 Madrid, Spain \\ ${ }^{31}$ Massachusetts Institute of Technology, Cambridge, Massachusetts 02139, USA \\ ${ }^{32}$ University of Michigan, Ann Arbor, Michigan 48109, USA \\ ${ }^{33}$ Michigan State University, East Lansing, Michigan 48824, USA \\ ${ }^{34}$ Institution for Theoretical and Experimental Physics, ITEP, Moscow 117259, Russia \\ ${ }^{35}$ University of New Mexico, Albuquerque, New Mexico 87131, USA \\ ${ }^{36}$ The Ohio State University, Columbus, Ohio 43210, USA \\ ${ }^{37}$ Okayama University, Okayama 700-8530, Japan \\ ${ }^{38}$ Osaka City University, Osaka 558-8585, Japan \\ ${ }^{39}$ University of Oxford, Oxford OX1 3RH, United Kingdom \\ ${ }^{40}$ Istituto Nazionale di Fisica Nucleare, Sezione di Padova, I-35131 Padova, Italy \\ ${ }^{41}$ University of Padova, I-35131 Padova, Italy \\ ${ }^{42}$ University of Pennsylvania, Philadelphia, Pennsylvania 19104, USA \\ ${ }^{43}$ Istituto Nazionale di Fisica Nucleare Pisa, I-56127 Pisa, Italy \\ ${ }^{44}$ University of Pisa, I-56127 Pisa, Italy \\ ${ }^{45}$ University of Siena, I-56127 Pisa, Italy \\ ${ }^{46}$ Scuola Normale Superiore, I-56127 Pisa, Italy
}




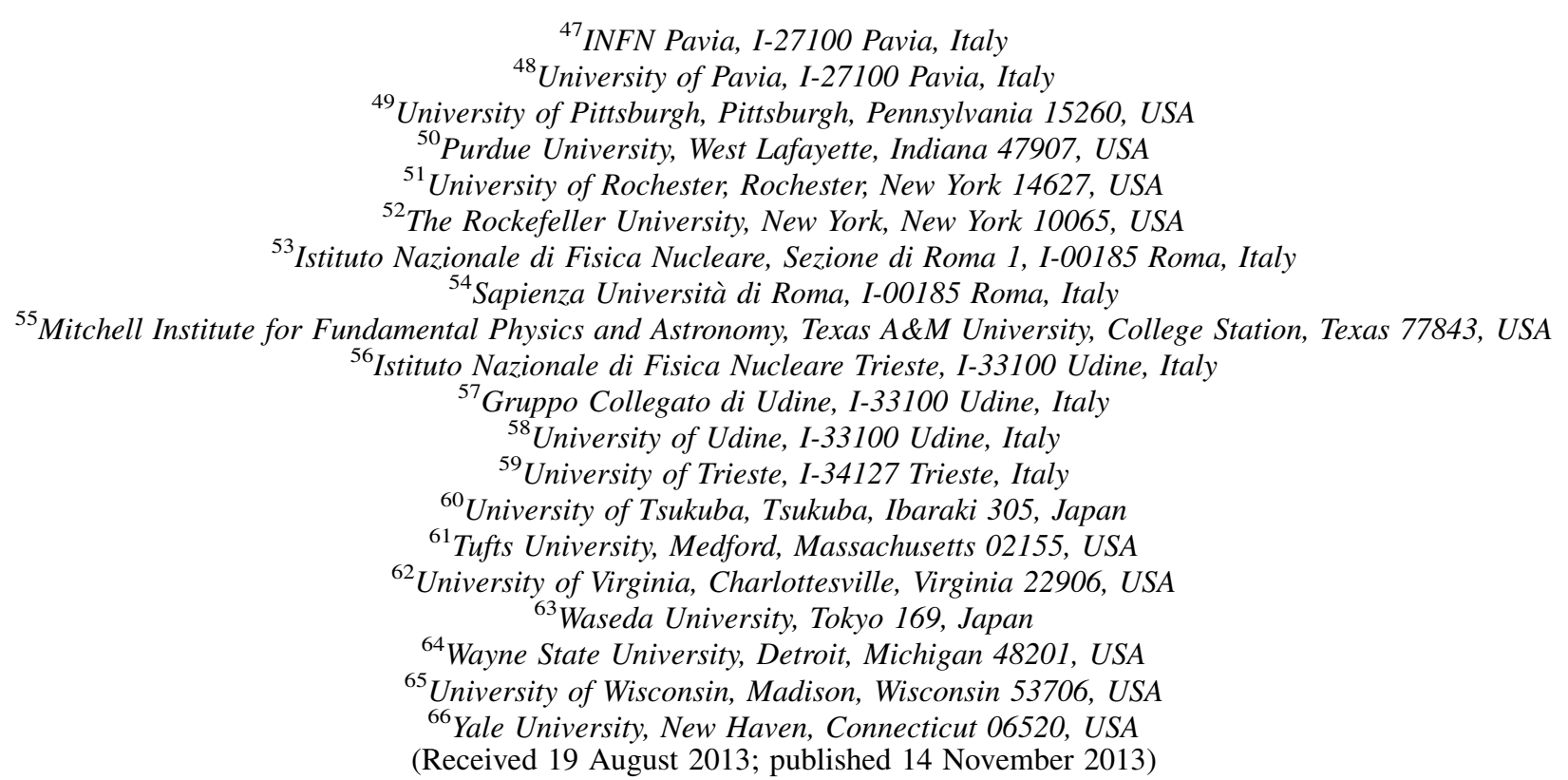

We present a measurement of the total decay width of the top quark using events with top-antitop quark pair candidates reconstructed in the final state with one charged lepton and four or more hadronic jets. We use the full Tevatron run II data set of $\sqrt{s}=1.96 \mathrm{TeV}$ proton-antiproton collisions recorded by the CDF II detector. The top quark mass and the mass of the hadronically decaying $W$ boson are reconstructed for each event and compared with distributions derived from simulated signal and background samples to extract the top quark width $\left(\Gamma_{\text {top }}\right)$ and the energy scale of the calorimeter jets with in situ calibration. For a top quark mass $M_{\text {top }}=172.5 \mathrm{GeV} / c^{2}$, we find $1.10<\Gamma_{\text {top }}<4.05 \mathrm{GeV}$ at $68 \%$ confidence level, which is in agreement with the standard model expectation of $1.3 \mathrm{GeV}$ and is the most precise direct measurement of the top quark width to date.

DOI: 10.1103/PhysRevLett.111.202001

PACS numbers: 14.65.Ha, 12.15.Ff, 13.85.Ni, 13.85.Qk

The top quark $(t)$ is the heaviest known elementary particle. Its large mass endows it with the largest decay width and, hence, the shortest lifetime of any of the known fermions [1]. At leading order calculation of quantum chromodynamics $(\mathrm{QCD})$, the top quark decay width $\left(\Gamma_{\text {top }}\right)$ depends on the top quark mass $\left(M_{\text {top }}\right)$, the Fermi coupling constant $\left(G_{F}\right)$, and the magnitude of the top-to-bottom quark coupling in the quark-mixing matrix $\left(\left|V_{t b}\right|\right)$ [2]. The next-to-leading-order calculation with QCD and electroweak corrections predicts $\Gamma_{\text {top }}=1.33 \mathrm{GeV}$ at $M_{\text {top }}=172.5 \mathrm{GeV} / c^{2}$ with approximately $1 \%$ precision $[3,4]$. This is consistent with the recent next-to-nextto-leading-order calculation of $\Gamma_{\text {top }}=1.32 \mathrm{GeV}$ [5]. A deviation from the standard model (SM) prediction could indicate the presence of non-SM decay channels, such as decays through a charged Higgs boson [6], the supersymmetric top quark partner [7], or a flavor-changing neutral current [8]. A direct measurement of $\Gamma_{\text {top }}$ provides general constraints on such processes.

The D0 Collaboration has determined the width to be $\Gamma_{\text {top }}=2.00_{-0.43}^{+0.47} \mathrm{GeV}$ in a data set corresponding to an integrated luminosity of $5.4 \mathrm{fb}^{-1}$, using a modeldependent, indirect measurement that assumes SM couplings [9]. The CDF Collaboration reported more model-independent measurements of the width using a direct shape comparison of the reconstructed top quark mass in data to the simulated top quark mass distributions $[10,11]$. The most recent measurement set an upper limit of $\Gamma_{\text {top }}<7.6 \mathrm{GeV}$ at the $95 \%$ confidence level (C.L.) with a data set corresponding to $4.3 \mathrm{fb}^{-1}$ [11]. Even though the direct measurement is less precise than the indirect one, it probes a broader class of non-SM physics models, because the direct measurement has less dependence on the SM.

This Letter reports on a direct measurement of the top quark width in $p \bar{p}$ collisions at the Tevatron, using the full run II data set, corresponding to an integrated luminosity of $8.7 \mathrm{fb}^{-1}$ collected with the CDF II detector [12], which is a general-purpose azimuthally and forward-backward symmetric detector surrounding the colliding beams of the Tevatron $p \bar{p}$ collider. We not only increase statistical sensitivity using a larger sample with respect to Ref. [11], but also improve jet-energy calibrations using an artificial neural network [13].

Top quarks at the Tevatron are predominantly produced in $t \bar{t}$ pairs. We reconstruct top quark decays in the topology 
of $t \rightarrow b W^{+}$and $\bar{t} \rightarrow \bar{b} W^{-}$. Events with a $W$ boson decaying into a charged lepton (electron or muon) and a neutrino [W $\rightarrow \ell \nu$ including the cascade decay of $W \rightarrow \tau(\rightarrow \ell \bar{\nu}) \nu]$ and the other $W$ boson decaying into a pair of jets (collimated sprays of particles resulting from the hadronization of quarks) define the lepton + jets channel $(t \bar{t} \rightarrow \ell \nu b \bar{b} q \bar{q})$. To select $t \bar{t}$ candidate events in this channel, we require one electron (muon) with $E_{T}>20 \mathrm{GeV}\left(p_{T}>20 \mathrm{GeV} / c\right)$ and pseudorapidity $|\eta|<1.1$ [14]. We also require large missing transverse energy [15] $\left(\mathbb{E}_{T}>20 \mathrm{GeV}\right)$ and at least four hadronic jets. Jets are reconstructed by combining signals from particles detected within a spatial cone of radius $\Delta R=\sqrt{(\Delta \eta)^{2}+(\Delta \phi)^{2}}=0.4$ [16]. Observed jet energies are corrected for nonuniformities of the calorimeter response parametrized as a function of $\eta$, the energy contributed by multiple $p \bar{p}$ interactions in the event, and the calorimeter's nonlinear response [17]. In addition to the standard jet-energy corrections, we use an artificial neural network that includes additional information, such as jet momentum from the charged particles inside the jet [13], to improve jet-energy resolution $[18,19]$. Jets originating from $b$ quarks are identified (tagged) using a secondary-vertex-tagging algorithm [20].

We divide the sample of $t \bar{t}$ candidates into subsamples with zero (0-tag), one (1-tag), and two or more (2-tag) $b$-tagged jets, which have different signal-to-background ratios. We further classify the events according to the jet kinematic properties. The "tight" selection requires exactly four jets, each with $E_{T}>20 \mathrm{GeV}$ and $|\eta|<2.0$. The "loose" selection on the remaining events requires exactly three jets with $E_{T}>20 \mathrm{GeV}$ and $|\eta|<2.0$, and one or more additional jets with $E_{T}>12 \mathrm{GeV}$ and $|\eta|<$ 2.4. We then combine the $b$-tag and jet-selection categories into five subsamples used in the analysis: 0-tagT, 1-tagL, 1-tagT, 2-tagL, and 2-tagT, where "T" and "L" denote the "tight" and "loose" jet selections. Finally, to reduce the level of non- $t \bar{t}$ background contributions to the 0-tag and 1-tag samples, we require the scalar sum of transverse energies in the event, $H_{T}=E_{T}^{\ell}+\not E_{T}+\sum_{\text {fourjets }} E_{T}^{\text {jet }}$, to exceed $250 \mathrm{GeV}$.

The primary sources of non- $t \bar{t}$ backgrounds are $W+$ jets and multijet production. We also consider small contributions from $Z+$ jets, dibosons, and single top quark production. The multijet background is estimated by the data-driven techniques described in Ref. [21]. The kinematic distributions of $W+$ jets are modeled with the ALPGEN [22] generator. The number of $W+$ jets events is determined from the total number of events observed in data by subtraction of the expected $t \bar{t}$ and the other background event contributions. Diboson backgrounds are modeled by ALPGEN for $W W, W Z, Z Z$ and PYTHIA [23] for $W \gamma$, while single top quark processes are generated with MADGRAPH [24]. We normalize simulated event yields using their theoretical next-to-leading-order cross sections [25]. References [20,26] provide the details of these techniques. Table I summarizes the sample composition in each subsample.

To distinguish between different values of $\Gamma_{\text {top }}$, we compare the reconstructed top quark mass distribution observed in data to various distributions from $t \bar{t}$ signal samples generated using PYTHIA with different $\Gamma_{\text {top }}$ values ranging from 0.1 to $30 \mathrm{GeV}$ for a fixed $M_{\text {top }}=$ $172.5 \mathrm{GeV} / c^{2}$. Because the jet-energy scale (JES) is one of the dominant systematic uncertainties in the analysis [10], we generate a set of samples where the JES is varied independently. In the data, jet energies are corrected to account for the energy scale error in the calorimeter with uncertainty $\sigma_{c}$ the CDF JES fractional uncertainty [17]. In the simulation, we vary the JES with the correction factor of jet energies, $1+\Delta_{\mathrm{JES}}$, with varying the values of $\Delta_{\mathrm{JES}}$ from $-3.0 \sigma_{c}$ to $+3.0 \sigma_{c}$.

After event selection, the analysis proceeds in three steps. First, we reconstruct a top quark mass $\left(m_{t}^{\text {reco }}\right)$, defined below, from each event. The width of the $m_{t}^{\text {reco }}$ distribution is a sensitive variable for $\Gamma_{\text {top. }}$. We also reconstruct the hadronically decaying $W$-boson mass $\left(m_{j j}\right)$. The constraint of $m_{j j}$ to the known $W$-boson mass can be used

TABLE I. Expected and observed numbers of signal and background events assuming a $t \bar{t}$ production cross section $\sigma_{t \bar{t}}=7.45 \mathrm{pb}$ and $M_{\text {top }}=172.5 \mathrm{GeV} / c^{2}$.

\begin{tabular}{|c|c|c|c|c|c|}
\hline & 0 -tag & 1-tagL & 1-tag T & 2-tagL & 2-tagT \\
\hline$W+$ jets & $703 \pm 199$ & $170 \pm 60$ & $102 \pm 37$ & $11.6 \pm 4.9$ & $8.4 \pm 3.5$ \\
\hline$Z+$ jets & $52.3 \pm 4.4$ & $8.9 \pm 1.1$ & $5.9 \pm 0.7$ & $0.8 \pm 0.1$ & $0.5 \pm 0.1$ \\
\hline Single top & $4.8 \pm 0.5$ & $10.5 \pm 0.9$ & $6.8 \pm 0.6$ & $2.2 \pm 0.3$ & $1.7 \pm 0.2$ \\
\hline Diboson & $60.3 \pm 5.6$ & $11.1 \pm 1.4$ & $8.5 \pm 1.1$ & $1.0 \pm 0.2$ & $0.8 \pm 0.1$ \\
\hline Multijets & $143 \pm 114$ & $34.5 \pm 12.6$ & $20.7 \pm 16.6$ & $4.4 \pm 2.5$ & $2.5 \pm 2.4$ \\
\hline Background & $963 \pm 229$ & $235 \pm 61$ & $144 \pm 41$ & $19.9 \pm 5.5$ & $13.8 \pm 4.2$ \\
\hline$t \bar{t}$ signal & $645 \pm 86$ & $695 \pm 87$ & $867 \pm 108$ & $192 \pm 30$ & $304 \pm 47$ \\
\hline Expected & $1608 \pm 245$ & $930 \pm 106$ & $1011 \pm 115$ & $212 \pm 30$ & $318 \pm 47$ \\
\hline Observed & 1627 & 882 & 997 & 208 & 275 \\
\hline
\end{tabular}


to determine the JES calibration in situ, which reduces the dominant uncertainty from the JES. The second step is a likelihood fit of $m_{t}^{\text {reco }}$ and $m_{j j}$ comparing with simulated signal and background distributions to determine $\Gamma_{\text {meas }}$, an estimator of $\Gamma_{\text {top }}$, which will be explained later. Finally, we use a likelihood-ratio ordering to determine the $68 \%$ and 95\% C.L. limits of $\Gamma_{\text {top }}$ from $\Gamma_{\text {meas }}$ [27].

For the event reconstruction, we assume that all selected events are lepton + jets $t \bar{t}$ events and perform a complete reconstruction of the $t \bar{t}$ kinematic properties [28,29]. We perform a $\chi^{2}$ minimization to fit the momenta of the $t \bar{t}$ decay products and determine $m_{t}^{\text {reco }}$ for each event using the four leading jets. To resolve the ambiguity arising from the jets-to-quarks assignments, we require that $b$-tagged jets are assigned to $b$ quarks and select the assignment with the lowest $\chi^{2}$. To reject events having poorly reconstructed kinematic properties, we request the minimum value of $\chi^{2}$ to be less than 9.0 (less than 3.0) for the $b$-tagged (zero $b$-tag) events. The dijet mass, $m_{j j}$, is calculated independently as the invariant mass of two non- $b$-tagged jets that provides the closest value to the known $W$-boson mass, $80.4 \mathrm{GeV} / c^{2}$ [30]. Figure 1(a) shows the distributions of $m_{t}^{\text {reco }}$ for three different $\Gamma_{\text {top }}$ values. The shape of $m_{t}^{\text {reco }}$ depends on $\Gamma_{\text {top }}$, yielding an estimate of its value. Distributions of $m_{j j}$ for three different values of $\Delta_{\mathrm{JES}}$ are shown in Fig. 1(b). The maximum of the distribution depends strongly on $\Delta_{\mathrm{JES}}$. Hence, $m_{j j}$ can be used to constrain the JES in situ.

To account for the correlation between $m_{t}^{\text {reco }}$ and $m_{j j}$, we construct two-dimensional probability density functions (PDFs) of signals and background with the twodimensional kernel-density estimates [31] for the likelihood fit procedure [29]. First, at discrete values of $\Gamma_{\text {top }}$ from 0.1 to $30 \mathrm{GeV} / c^{2}$ and $\Delta_{\mathrm{JES}}$ from $-3.0 \sigma_{c}$ to $+3.0 \sigma_{c}$, we estimate the PDFs for the observables from the abovementioned PYTHIA $t \bar{t}$ samples. Background PDFs are estimated for various values of $\Delta_{\mathrm{JES}}$ from $-3.0 \sigma_{c}$ to $+3.0 \sigma_{c}$. We interpolate the simulated distributions to find PDFs for arbitrary values of $\Gamma_{\text {top }}$ and $\Delta_{\mathrm{JES}}$ using a local polynomial smoothing method [32]. Then, we fit the signal and background PDFs to the unbinned distributions observed in the data. In the fit of the data, we apply a Gaussian constraint to the expected number of background events, but there are no constraints on the expected number of signal events. Separate likelihoods are constructed for the five subsamples, and the overall likelihood is obtained by multiplying them together. Maximization of the total likelihood yields the best-fit value $\Gamma_{\text {meas }}$.

The limit on the true value of $\Gamma_{\text {top }}$ from the measured $\Gamma_{\text {meas }}$ is set using the Neyman construction [33]. In this procedure, the unphysical region of negative $\Gamma_{\text {top }}$ is not allowed for $\Gamma_{\text {meas }}$, which makes the acceptance region of $\Gamma_{\text {meas }}$ to be equal or greater than zero. It makes the large number of events at $\Gamma_{\text {meas }}$ equal to zero for a small $\Gamma_{\text {top }}$. We derive the confidence bands from simulated experiments in which signal and background events are selected from the simulated samples.

We examine various sources of systematic uncertainties that could effect the $\Gamma_{\text {top }}$ measurement. Because this measurement relies on the shape of $m_{t}^{\text {reco }}$, the uncertainties on the JES calibration and the jet resolution could dominate. However, the JES is well controlled with in situ calibration using the $m_{j j}$ distributions. To estimate the uncertainty from the jet-energy resolution, we use experimental and simulated data samples of events with a photon recoiling against a jet in the final state. In these samples, we estimate the energy of the jets using the energy of the recoiled photon. We compare the $p_{T}$-dependent resolutions on the energy of the reconstructed jets in data and simulation. We obtain consistent results within statistical uncertainty. Taking into account statistical uncertainty of the data, we define a $p_{T}$-dependent systematic uncertainty on jet

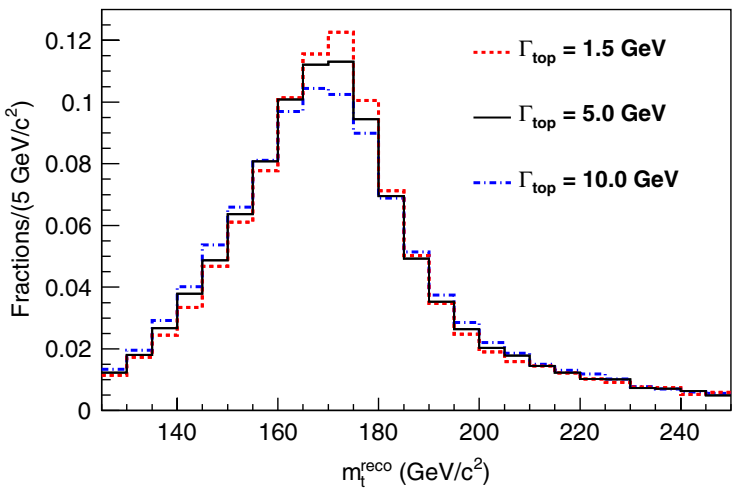

(a)

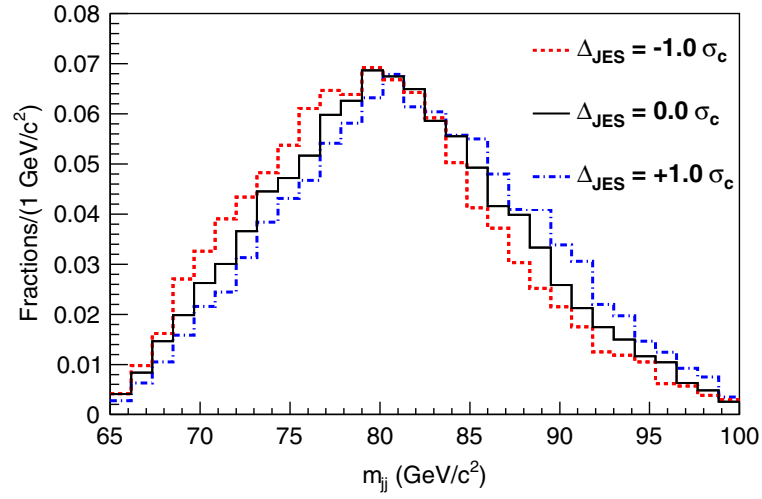

(b)

FIG. 1 (color online). Distributions for simulated events meeting the lepton + jets selection: (a) $m_{t}^{\text {reco }}$ distributions displayed with three values of $\Gamma_{\text {top }}$ and with the nominal $\Delta_{\mathrm{JES}}=0.0$, (b) $m_{j j}$ distributions displayed with three values of $\Delta_{\mathrm{JES}}$ and with $\Gamma_{\text {top }}=1.5 \mathrm{GeV}$. 
TABLE II. Summary of systematic uncertainties on $\Gamma_{\text {top }}$.

\begin{tabular}{lc}
\hline \hline Source & Uncertainty $(\mathrm{GeV})$ \\
\hline Jet resolution & 0.56 \\
Color reconnection & 0.69 \\
Event generator & 0.50 \\
Higher-order effects & 0.21 \\
Residual jet-energy scale & 0.19 \\
Parton distribution functions & 0.24 \\
$b$-jet energy scale & 0.28 \\
Background shape & 0.18 \\
Gluon fusion fraction & 0.26 \\
Initial- and final-state radiation & 0.17 \\
Lepton energy scale & 0.03 \\
Multiple hadron interaction & 0.23 \\
Total systematic uncertainty & 1.22 \\
\hline \hline
\end{tabular}

resolution to cover the difference. In addition to the jetenergy resolution, the uncertainties associated with modeling of color flow in the interaction and with the arbitrary choice of the event generator are the dominant systematic uncertainties, as shown in Table II. The color-reconnection systematic uncertainty takes into account the effects of the underlying color structure of quarks and gluons and its flow [34] by rearrangements from the simplest configuration to enhanced color reconnections based on simulations with differently tuned configuration parameters [35]. For the systematic uncertainty associated with the choice of the event generator, the samples generated by PYTHIA and HERWIG [36] are used. We examine the effects of higher-order corrections using MC@NLO [37], a full next-to-leading-order simulation. Other sources of systematic effects, including uncertainties in parton-distribution functions, initial- and final-state gluon radiation, multiple hadron interactions, $b$-jet-energy scale, gluon fusion fraction, background shape, and lepton-energy scale, give small contributions. The total systematic uncertainty of $1.22 \mathrm{GeV}$ is calculated as a quadrature sum of the listed uncertainties. We estimate the systematic uncertainties under the assumptions of $M_{\text {top }}=172.5 \mathrm{GeV} / c^{2}$ and $\Gamma_{\text {top }}=1.5 \mathrm{GeV} / c^{2}$, but checks with different values of $M_{\text {top }}$ and $\Gamma_{\text {top }}$ for the dominant sources show consistent results. The details of the systematic-uncertainty evaluations are described in Refs. [28,29,38].

To incorporate systematic effects into the confidence bands we use a convolution method for folding systematic effects into the likelihood function $[39,40]$ based on Bayesian treatment of systematic uncertainties [41,42]. We convolve the likelihood function with a Gaussian PDF that has a width equal to $1.22 \mathrm{GeV}$ and is centered at zero. We then build the confidence bands with $68 \%$ and $95 \%$ coverages as shown in Fig. 2. The value of $\Gamma_{\text {meas }}$ retrieved from the data is $1.63 \mathrm{GeV}$ and is depicted as an arrow in the plot. This corresponds to an upper limit of $\Gamma_{\text {top }}<6.38 \mathrm{GeV}$ at the $95 \%$ C.L. We also set a

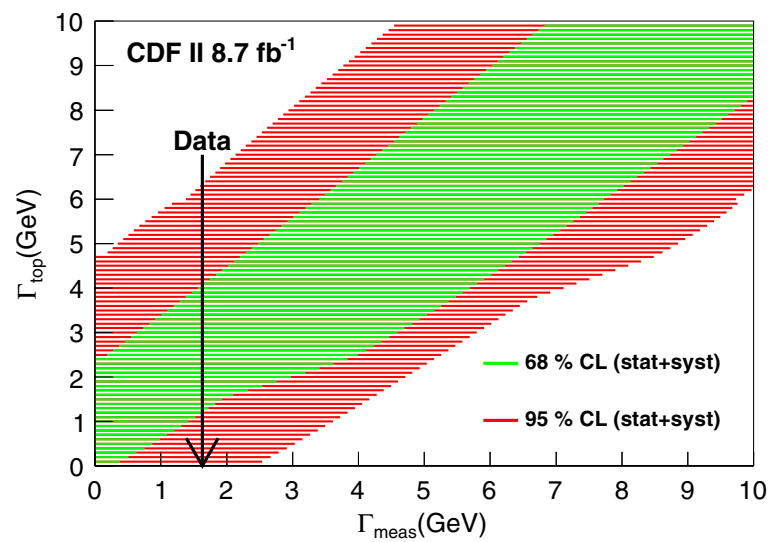

FIG. 2 (color online). Confidence bands of $\Gamma_{\text {top }}$ as a function of $\Gamma_{\text {meas }}$ for $68 \%$ and $95 \%$ C.L. limits. Results from simulated experiments assuming $8.7 \mathrm{fb}^{-1}$ of data at different values of $\Gamma_{\text {top }}$ are convoluted with a smearing function to account for systematic uncertainties. The value observed in data is indicated by an arrow.

two-sided limit of $1.10<\Gamma_{\text {top }}<4.05 \mathrm{GeV}$ at the $68 \%$ C.L., which corresponds to a lifetime of $1.6 \times$ $10^{-25}<\tau_{\text {top }}<6.0 \times 10^{-25}$ s. For a typical quark hadronization time scale, $3.3 \times 10^{-24} \mathrm{~s}$ [43], this result supports the assertion that top quark decay occurs before hadronization.

In conclusion, a direct measurement of the top quark width is performed in fully reconstructed lepton + jets events by using the full CDF run II data set corresponding to an integrated luminosity of $8.7 \mathrm{fb}^{-1}$ of $p \bar{p}$ collisions at $\sqrt{s}=$ $1.96 \mathrm{TeV}$. We obtain $1.10<\Gamma_{\text {top }}<4.05 \mathrm{GeV}$ at $68 \%$ C.L., which corresponds to a lifetime of $1.6 \times 10^{-25}<\tau_{\text {top }}<$ $6.0 \times 10^{-25} \mathrm{~s}$. This is the most precise direct determination of the top quark width and lifetime and shows no evidence of non-SM physics in the top quark decay.

We thank the Fermilab staff and the technical staffs of the participating institutions for their vital contributions. This work was supported by the U.S. Department of Energy and National Science Foundation; the Italian Istituto Nazionale di Fisica Nucleare; the Ministry of Education, Culture, Sports, Science and Technology of Japan; the Natural Sciences and Engineering Research Council of Canada; the National Science Council of the Republic of China; the Swiss National Science Foundation; the A.P. Sloan Foundation; the Bundesministerium für Bildung und Forschung, Germany; the Korean World Class University Program, the National Research Foundation of Korea; the Science and Technology Facilities Council and the Royal Society, UK; the Russian Foundation for Basic Research; the Ministerio de Ciencia e Innovación, and Programa Consolider-Ingenio 2010, Spain; the Slovak R\&D Agency; the Academy of Finland; the Australian Research Council (ARC); and the EU community Marie Curie Fellowship Contract No. 302103. 
${ }^{\mathrm{a}}$ Deceased.

${ }^{\mathrm{b}}$ Visitor from University of British Columbia, Vancouver, BC V6T 1Z1, Canada.

${ }^{\mathrm{c}}$ Visitor from Istituto Nazionale di Fisica Nucleare, Sezione di Cagliari, 09042 Monserrato (Cagliari), Italy.

${ }^{\mathrm{d}}$ Visitor from University of California Irvine, Irvine, CA 92697, USA.

${ }^{\mathrm{e}}$ Visitor from Institute of Physics, Academy of Sciences of the Czech Republic, 182 21, Czech Republic.

${ }^{\mathrm{f}}$ Visitor from CERN, CH-1211 Geneva, Switzerland.

${ }^{\mathrm{g}}$ Visitor from Cornell University, Ithaca, NY 14853, USA.

${ }^{\mathrm{h}}$ Visitor from University of Cyprus, Nicosia CY-1678, Cyprus.

${ }^{\mathrm{i}}$ Visitor from Office of Science, U.S. Department of Energy, Washington, DC 20585, USA.

${ }^{\mathrm{j} V i s i t o r ~ f r o m ~ U n i v e r s i t y ~ C o l l e g e ~ D u b l i n, ~ D u b l i n ~ 4, ~ I r e l a n d . ~}$

${ }^{\mathrm{k}}$ Visitor from ETH, 8092 Zürich, Switzerland.

${ }^{\mathrm{l} V i s i t o r}$ from University of Fukui, Fukui City, Fukui Prefecture, Japan 910-0017.

${ }^{\mathrm{m}}$ Visitor from Universidad Iberoamericana, Lomas de Santa Fe, México, C.P. 01219, Distrito Federal.

${ }^{\mathrm{n}}$ Visitor from University of Iowa, Iowa City, IA 52242, USA.

${ }^{\circ}$ Visitor from Kinki University, Higashi-Osaka City, Japan 577-8502.

${ }^{\mathrm{p}}$ Visitor from Kansas State University, Manhattan, KS 66506, USA.

${ }^{\mathrm{q}}$ Visitor from Brookhaven National Laboratory, Upton, NY 11973, USA.

${ }^{\mathrm{r}}$ Visitor from Queen Mary, University of London, London, E1 4NS, United Kingdom.

${ }^{\mathrm{s}}$ Visitor from University of Melbourne, Victoria 3010, Australia.

${ }^{t}$ Visitor from Muons, Inc., Batavia, IL 60510, USA.

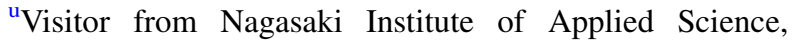
Nagasaki 851-0193, Japan.

${ }^{v}$ Visitor from National Research Nuclear University, Moscow 115409, Russia.

${ }^{\mathrm{w}}$ Visitor from Northwestern University, Evanston, IL 60208, USA.

${ }^{\mathrm{x}}$ Visitor from University of Notre Dame, Notre Dame, IN 46556, USA.

${ }^{\mathrm{y}}$ Visitor from Universidad de Oviedo, E-33007 Oviedo, Spain.

${ }^{\mathrm{z}}$ Visitor from CNRS-IN2P3, Paris, F-75205 France.

${ }^{\text {aa } V i s i t o r ~ f r o m ~ U n i v e r s i d a d ~ T e c n i c a ~ F e d e r i c o ~ S a n t a ~ M a r i a, ~}$ 110v Valparaiso, Chile.

${ }^{\mathrm{bb}}$ Visitor from The University of Jordan, Amman 11942, Jordan.

${ }^{\mathrm{cc}}$ Visitor from Universite catholique de Louvain, 1348 Louvain-La-Neuve, Belgium.

${ }^{\text {dd } V i s i t o r ~ f r o m ~ U n i v e r s i t y ~ o f ~ Z u ̈ r i c h, ~} 8006$ Zürich, Switzerland.

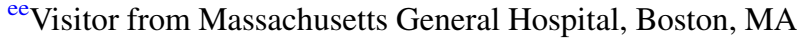
02114, USA.

${ }^{\mathrm{ff}}$ Visitor from Harvard Medical School, Boston, MA 02114, USA.
${ }^{\mathrm{gg}}$ Visitor from Hampton University, Hampton, VA 23668, USA.

${ }^{\text {hh }}$ Visitor from Los Alamos National Laboratory, Los Alamos, NM 87544, USA.

${ }^{i i}$ Visitor from Università degli Studi di Napoli Federico I, I-80138 Napoli, Italy.

[1] J. Beringer et al. (Particle Data Group), Phys. Rev. D 86, 010001 (2012).

[2] A. Denner and T. Sack, Nucl. Phys. B358, 46 (1991).

[3] A. Czarnecki and K. Melnikov, Nucl. Phys. B544, 520 (1999).

[4] K. G. Chetyrkin, R. Harlander, T. Seidensticker, and M. Steinhauser, Phys. Rev. D 60, 114015 (1999).

[5] J. Gao, C.S. Li, and H.X. Zhu, Phys. Rev. Lett. 110, 042001 (2013).

[6] V. Barger, J. L. Hewett, and R. J. N. Phillips, Phys. Rev. D 41, 3421 (1990).

[7] K. I. Hikasa and M. Kobayashi, Phys. Rev. D 36, 724 (1987); C. S. Li, J. M. Yang, and B. Q. Hu, Phys. Rev. D 48, 5425 (1993).

[8] J. L. Diaz-Cruz, M. A. Perez, G. Tavares-Velasco, and J. J. Toscano, Phys. Rev. D 60, 115014 (1999).

[9] V. M. Abazov et al. (D0 Collaboration), Phys. Rev. D 85, 091104 (2012).

[10] T. Aaltonen et al. (CDF Collaboration), Phys. Rev. Lett. 102, 042001 (2009).

[11] T. Aaltonen et al. (CDF Collaboration), Phys. Rev. Lett. 105, 232003 (2010).

[12] T. Aaltonen et al. (CDF Collaboration), Phys. Rev. D 71, 032001 (2005).

[13] T. Aaltonen, A. Buzatu, B. Kilminster, Y. Nagai, and W. Yao, arXiv:1107.3026.

[14] We use a right-handed spherical coordinate system with the origin at the center of the detector. The angles $\theta$ and $\phi$ are the polar and azimuthal angles, respectively, and $\theta$ is zero along incident proton direction. The pseudorapidity is defined by $\eta=-\ln \tan (\theta / 2)$. The transverse momentum and energy are defined by $p_{T}=p \sin (\theta)$ and $E_{T}=$ $E \sin (\theta)$, respectively, where $p$ and $E$ are the momentum and energy of the particle.

[15] The missing transverse energy, an imbalance of energy in the transverse plane of the detector, is defined by $\mathbb{E}_{T}=$ $-\left|\sum_{\text {towers }} E_{T} \hat{n}_{T}\right|$, where $\hat{n}_{T}$ is the unit vector normal to the beam and pointing to a given calorimeter tower and $E_{T}$ is the transverse energy measured in that tower.

[16] F. Abe et al. (CDF Collaboration), Phys. Rev. D 45, 1448 (1992).

[17] A. Bhatti et al., Nucl. Instrum. Methods Phys. Res., Sect. A 566, 375 (2006).

[18] T. Aaltonen et al. (CDF Collaboration), Phys. Rev. Lett. 109, 152003 (2012).

[19] T. Aaltonen et al. (CDF Collaboration), Phys. Rev. D 87, 052013 (2013).

[20] D. Acosta et al. (CDF Collaboration), Phys. Rev. D 71, 052003 (2005).

[21] T. Aaltonen et al. (CDF Collaboration), Phys. Rev. D 77, 011108 (2008).

[22] M. L. Mangano, F. Piccinini, A. D. Polosa, M. Moretti, and R. Pittau, J. High Energy Phys. 07 (2003) 001.

[23] T. Sjöstrand, S. Mrenna, and P. Skands, J. High Energy Phys. 05 (2006) 026 
[24] J. Alwall, P. Demin, S. D. Visscher, R. Frederix, M. Herquet, F. Maltoni, T. Plehn, D. L. Rainwater, and T. Stelzer, J. High Energy Phys. 09 (2007) 028.

[25] J. M. Campbell and R. K. Ellis, Phys. Rev. D 60, 113006 (1999); B. W. Harris, E. Laenen, L. Phaf, Z. Sullivan, and S. Weinzierl, Phys. Rev. D 66, 054024 (2002).

[26] T. Aaltonen et al. (CDF Collaboration), Phys. Rev. Lett. 105, 012001 (2010).

[27] G. J. Feldman and R. D. Cousins, Phys. Rev. D 57, 3873 (1998).

[28] T. Aaltonen et al. (CDF Collaboration), Phys. Rev. D 73, 032003 (2006).

[29] T. Aaltonen et al. (CDF Collaboration), Phys. Rev. D 79, 092005 (2009).

[30] T. Aaltonen et al. (CDF Collaboration), Phys. Rev. Lett. 108, 151803 (2012); V.M. Abazov el al. (D0 Collaboration), Phys. Rev. Lett. 108, 151804 (2012).

[31] K. Cranmer, Comput. Phys. Commun. 136, 198 (2001).

[32] C. Loader, Local Regression and Likelihood (Springer, New York, 1999).

[33] J. Neyman, Phil. Trans. R. Soc. A 236, 333 (1937).

[34] P.Z. Skands and D. Wicke, Eur. Phys. J. C 52, 133 (2007).
[35] P.Z. Skands, Phys. Rev. D 82, 074018 (2010).

[36] G. Corcella, I. G. Knowles, G. Marchesini, S. Moretti, K. Odagiri, P. Richardson, M. H. Seymour, and B. R. Webber, J. High Energy Phys. 01 (2001) 010.

[37] S. Frixione and B. R. Webber, J. High Energy Phys. 06 (2002) 029.

[38] T. Aaltonen et al. (CDF and D0 Collaborationa), Phys. Rev. D 86, 092003 (2012).

[39] J. O. Berger, B. Liseo, and R. L. Wolpert, Stat. Sci. 14, 1 (1999).

[40] L. Demortier, Proceedings of the Advanced Statistical Techniques in Particle Physics, Durham, UK, 2002, edited by M.R. Whalley and L. Lyons (Durham University, Durham, UK, 2002), p. 145, IPPP/02/39.

[41] J. Conrad, O. Botner, A. Hallgren, and C. Perez de los Heros, Phys. Rev. D 67, 012002 (2003); G. C. Hill, Phys. Rev. D 67, 118101 (2003).

[42] F. Tegenfeldt and J. Conrad, Nucl. Instrum. Methods Phys. Res., Sect. A 539, 407 (2005).

[43] I. Bigi, Y. Dokshitzer, V. Khoze, J. Kuhn, and P. Zerwas, Phys. Lett. B 181, 157 (1986); L. H. Orr and J. L. Rosner, Phys. Lett. B 246, 221 (1990). 